\title{
Breastfeeding: A Clinical Imperative
}

\author{
Michelle G. Brenner, M.D., IBCLC, and E. Stephen Buescher, M.D.
}

\begin{abstract}
Hospital breastfeeding initiation rates (75\%) show that most mothers in the United States want to breastfeed and are trying to do so. Even from the very start, however, mothers may not be getting the breastfeeding support they need. Low breastfeeding rates at 3, 6, and 12 months illustrate that women face multiple additional barriers to maintaining breastfeeding. What can we do to help more mothers be more successful? As healthcare providers, we need to be believers that breastfeeding is worth the effort. Perhaps most important for us is to realize that human milk is not simply a food but rather a complex, human infant support system. We can then articulate to families the importance of breastfeeding as a clinical imperative, a preventer of acute and chronic illness and disease.

It will take integration, normalization, and mainstreaming of breastfeeding into our culture for acceptance and growth of the practice. Once we assist families in making educated decisions about breastfeeding, we need to provide supportive environments in our hospitals, medical practices, workplaces, and communities that implement the best ways to support breastfeeding. Breast milk is worth the effort, and the time has come to be ardent supporters of mothers and infants and their breastfeeding intentions.
\end{abstract}

\section{Introduction}

B REASTFEEDING RATES IN the United States have continued to slowly creep upward. Current estimates suggest approximately $75 \%$ of women initiate breastfeeding in the hospital, meeting the Healthy People 2010 goal for initiation of breastfeeding. However, only $43 \%$ of women continue to breastfeed through 6 months, and 22\% through 12 months, falling short of the Healthy People 2010 targets. ${ }^{1}$ Looking toward the Healthy People 2020 goals (Table 1) leads us to the question: What do we need to do differently to get more women to breastfeed? We would propose that part of the answer is to get healthcare workers to be stronger supporters and more involved in helping mothers breastfeed. Rather than perceiving and presenting it as a desirable option for infant feeding, healthcare workers should see breastfeeding as a clinical imperative, no different from control of blood pressure in a hypertensive patient or vaccination of children against common childhood diseases.

So, what do healthcare workers need to understand to move them away from being politely supportive of breastfeeding to becoming advocates for it as a clinical imperative? Perhaps most important is to realize that human milk is not simply a food. Rather, it is a complex, sophisticated, and highly integrated human infant support system that provides the infant with protection, information, and nutrition. Its ability to provide this support to the infant results from its provision of both nonnutritive and food/nutritive compo- nents to the breastfed infant. The nonnutritive components in human milk include a significant portion of its contained lactose (approximately $50 \%-55 \%$ of breast milk by biochemical composition), essentially all of the nonlactose oligosaccharides (approximately 10\% of total composition), and various portions of the fat, protein, and ash fraction of human milk. They are responsible for many of the beneficial effects of human milk feeding, such as protection against many classes of enteric and upper aerodigestive tract pathogens and imparting of information to the nursing infant via volatile substances and flavors in the milk. Many of these effects are mediated via novel mechanisms that are best appreciated on the conceptual background of milk as a human infant support system.

\section{Protective Components-To Baby and Mother}

Of the benefits derived from breastfeeding, protection against infection is the most recognized and best scientifically documented outcome. It should be noted that modern prevention and medical care of infectious diseases tend to be organism specific. For example, influenza vaccination protects against influenza but not against other respiratory virus or bacterial pathogens, and antibiotic therapies can target classes of bacterial pathogens but have no effects on viral or protozoal pathogens. In comparison, human milk feeding is clearly protective against enteric infections caused by multiple bacterial, protozoal, and viral pathogens, ${ }^{2-6}$ against upper 
Table 1. U.S. Department of Health and Human Services Healthy People: Summary Goals and Statistics

\begin{tabular}{lccc}
\hline $\begin{array}{l}\text { Increase proportion } \\
\text { of infants } \\
\text { who are breastfed }\end{array}$ & $\begin{array}{c}2007-2009 \\
\text { baseline } \\
(\%)\end{array}$ & $\begin{array}{c}\text { Healthy People } \\
\text { 2010 target } \\
(\%)\end{array}$ & $\begin{array}{c}\text { Healthy People } \\
2020 \text { target } \\
(\%)\end{array}$ \\
\hline Ever & 73.9 & 75 & 81.9 \\
At 6 months & 43.4 & 50 & 60.6 \\
At 12 months & 22.7 & 25 & 34.1 \\
$\begin{array}{l}\text { Exclusive through } \\
\quad 3 \text { months }\end{array}$ & 33.1 & 40 & 46.2 \\
$\begin{array}{l}\text { Exclusive through } \\
\quad \text { 6 months }\end{array}$ & 13.6 & 17 & \\
\hline
\end{tabular}

Available at www.healthypeople.gov/2020/topicsobjectives2020/ objectiveslist.aspx?topicid $=26$

aerodigestive tract infections caused by bacteria and viruses, 7,8 and even against bacterial urinary tract infections (UTIs). ${ }^{9}$ This leads to the question: How can a single agent, human milk, be protective against multiple types of infections caused by multiple classes of pathogens at multiple sites mediated via multiple different pathophysiologies? The answer comes from the support system aspect alluded to above: protection is provided directly and indirectly via multiple milk components and activities that include immunoglobulins, various glycoproteins such as mucins and lactadherin, oligosaccharides, binding proteins and enzymatic activities, antioxidants, soluble cytokine receptors/cytokine antagonists, free fatty acids, acidic fecal $\mathrm{pH}$, and a characteristic fecal flora.

Direct binding effects, which aggregate potential pathogens/toxins and prevent disease by preventing their attachment to host target tissue, are mediated by secretory immunoglobulin A (IgA), oligosaccharides, and even milk fat globule membranes. ${ }^{10}$ Notably, by minimizing attachment/ infection potential via organism binding rather than overt organism killing (as occurs in classical human immunity), a pertinent point can be made: recovery of live pathogens from asymptomatic breastfed infants would be expected to occur, ${ }^{11}$ and symptom severity for a given type of infection may be minimized. The latter effect may also be affected by the anti-inflammatory components contained in human milk. ${ }^{12}$ Indirect effects of nonnutritive components mediating protection from infection relate to unexpected, nonhuman consumers of the lactose and oligosaccharides in human milkthe colonic microbial flora that occurs in the breastfed infant. This acid-producing, fermentative flora likely controls the numbers of potentially pathogenic adult enteric flora in the breastfed infant. Recent sequencing of the genome of Bifidobacterium longum subsp. infantis, clearly illustrates that this organism is metabolically optimized to use human milk carbohydrates as an energy source, ${ }^{13}$ reinforcing appreciation of the interactions and impacts of this infant support system.

It would be incorrect to presume that breastfeeding and human milk benefit the infants only via effects that counter/ prevent infection. The meta-analysis summary from the Alliance for Healthcare Research and Quality (AHRQ) makes the point clearly that breastfeeding decreases adult obesity as well as adult-onset type 2 diabetes mellitus. ${ }^{14}$ Although these effects are less well understood mechanistically, the infant support system standpoint might suggest that human milk feeding has long-range effects on metabolic efficiency and energy balance. In addition, whereas benefits to infants are easy to recount, the health benefits to mothers cannot be underestimated. Table 2 lists the cancer and chronic disease protection afforded to women and infants by breastfeeding. For both, it is important to note that cumulative breastmilk intake by an infant and cumulative breastfeeding experiences by the mother increase the overall protective effect of breastfeeding.

\section{Human Milk and the Breast as an Information Source}

All mammalian mothers feed milk to their young. Therefore, all mammalian young must also reach the stage of weaning. How mammalian mothers instruct their young as to what they should and should not consume when weaned was first suggested only a decade ago, largely based on studies of food acceptance in human infants at weaning. ${ }^{16}$ These studies have shown that maternal diet can influence the recognition and acceptance of foods by the infant at weaning, presumably via flavors or smells of their mother's milk. This effect likely helps the young mammal transition to a safe diet at weaning, but for humans, it also may have social connotations through facilitating acceptance of the foods associated with one's culture. Mother-infant information exchange via scents/ volatile compounds of the breast and breast milk is also a component of breastfeeding. Volatile components of areolar secretions from the glands of Montgomery have immediate effects on infants, producing mouthing and oral movements ${ }^{17}$ : the package is involved in the process! Volatile compounds from human milk or the breast help the infant locate the nipple ${ }^{18}$ and exert calming effects on the infant. ${ }^{19}$ There is no existing evidence that like other mammals, humans produce social chemosignals that affect human sexual motivation or reproductive behavior. However, a recent study of women exposed to "breastfeeding compounds" collected on pads worn next to the axillae and breasts of lactating women, suggest the existence of human pheromones. This is the first study to document the potential effect of natural human compounds, specifically those from nursing women and their infants, on the sexual motivation of other women. ${ }^{20} \mathrm{It}$, therefore, appears that as part of the support system, breastfeeding informs the infant about where the milk is now, triggers responses that facilitate the infant's obtaining milk, informs the infant of what foods are appropriate after weaning, and even extends its influences to other potential mothers.

\section{Cost Savings}

While the protective effects and information exchange aspects of human milk and breastfeeding are mechanistically

Table 2. Breastfeeding and Health Outcomes

\begin{tabular}{ll}
\hline $\begin{array}{l}\text { Decreased incidence } \\
\text { for women }\end{array}$ & \multicolumn{1}{c}{$\begin{array}{c}\text { Decreased incidence } \\
\text { for infants }\end{array}$} \\
\hline Breast cancer & Acute otitis media \\
Hyperlipidemia & Atopic dermatitis and asthma \\
Hypertension & Gastrointestinal illness \\
Ovarian cancer & Lower respiratory tract infections \\
Type 2 diabetes mellitus & Type 2 diabetes mellitus
\end{tabular}

Ip et al., 2007. ${ }^{14}$

Schwarz et al., 2009. ${ }^{15}$ 
sophisticated and intellectually curious, in the modern world, decisions often boil down to the answers to such questions as: What's in it for me? or What'll it cost me? The answers to such questions as they relate to breastfeeding can be summed up in the statement: It'll save you money. A series of studies, performed by government agencies and independent investigators, has documented the savings associated with breastfeeding from a variety of perspectives. From the population perspective of overall costs of illness in 2001, ${ }^{21}$ the impact (on three diseases) of raising the breastfeeding rate to the Healthy People 2010 targets (75\% initiating, 50\% at 6 months) was estimated to be a savings of $\$ 3.6$ billion. A more recent population-based assessment that examined effects on ten diseases estimates savings of $\$ 10-13$ billion per year if $80 \%-90 \%$ of families would exclusively breastfeed for 6 months. ${ }^{22}$ Although these monetary savings at the U.S. population level are the most striking, they are difficult to appreciate at the personal level. When healthcare use by exclusively formula-fed infants for lower respiratory tract infection, otitis media, and gastroenteritis was compared to that of exclusively or partially breastfed infants, both exclusive and partial breastfeeding decreased the numbers of office visits, follow-up visits, medications, and hospitalizations for all three illnesses. ${ }^{23}$ The authors then translated the calculated use of healthcare into the healthcare cost of formula feeding and estimated that these costs per 1000 formula-fed infants were $\$ 331,051$, or about $\$ 331$ per baby per year. For households that purchase formula at retail cost, a year's worth of powdered formula costs approximately $\$ 820$ in year 2000 prices. $^{24}$

The implications of the cost of formula itself and savings related to less infant healthcare use are blunted in populations that have access to deeply discounted formula costs or subsidized healthcare costs. The federal government purchases over half of all formula sold in the United States for food aid programs that supply formula at deeply discounted prices, ${ }^{25}$ and the populations using these programs typically also receive subsidized medical care. In these populations, enthusiastic encouragement and support of breastfeeding by healthcare providers likely has more impact than the specific economic considerations.

So, from multiple different perspectives, it is clear that breast is best-but how do we get there from here?

\section{Talk It Up!}

Women make feeding choices for their infants based on many influences, and these decisions are typically made well in advance of the birth of their baby. In one study, $78 \%$ of women decided their infant feeding plan before conception or during the first trimester of their pregnancy. ${ }^{26}$ This knowledge encourages us to discuss breastfeeding during preconception well-woman visits, perhaps in conjunction with teaching breast self-examination as a way to reduce one's risk of breast cancer and other diseases (Table 2). In caring for the newly pregnant woman, prenatal providers should take the opportunity to engage in a breastfeeding conversation early, simply endorsing it and recommending additional educational opportunities. Teachable moments about the benefits of breastfeeding should include the people who make a difference, the father and grandmothers. ${ }^{26,27}$ Kessler et al. ${ }^{27}$ reported $71 \%$ of feeding decisions were influenced by the infant's father and $29 \%$ by the maternal grandmother. A mother's primary sources of feeding information have been shown to be family $(33.9 \%)$, friends $(9.9 \%)$, the physician $(8.3 \%)$, prenatal class $(8.3 \%)$, and nurses in the hospital $(6.6 \%){ }^{26}$

Integrating, normalizing, and mainstreaming breastfeeding behavior into our culture is important for acceptance and growth of the practice. Whereas female breasts are routinely seen in the media as symbols of femininity and sexuality, they are infrequently used to portray breastfeeding as a common daily practice in movies, sit-coms, or advertisements. Best-forBabes is an example of a nonprofit organization designed to "give breastfeeding a makeover" and "beat the booby trapsthe barriers to breastfeeding success." 28 This novel social marketing approach on behalf of Mother Nature highlights examples of modern breastfeeding women in the media that might be emulated by women who follow pop culture. The goal is to empower women to make educated decisions and seek out the providers/facilities that will help them be most successful in attaining their goal to breastfeed. It will take many years of routine, daily, brief encounters with breastfeeding before it is thought of as the norm and gold standard for infant feeding.

\section{Breastfeeding Education for Mothers Improves Rates}

The U.S. Preventative Services Task Force identified education on breastfeeding as the most effective single intervention for increasing breastfeeding initiation and short-term duration. ${ }^{29}$ Cochrane Reviews also cite increased breast feeding rates when breastfeeding education was provided vs. controls. Cochrane's summary of best education practices notes educational information is effective in the prenatal and postnatal periods and is most effective when it is maternal need based, informal, and repetitious, focusing on interactions between mothers (peer support) before and after delivery. ${ }^{30}$ A breastfeeding class, taken alongside the baby's father, future grandmother, or girlfriend, is one way to learn the basics and have an additional breastfeeding advocate after the delivery. A postpartum breastfeeding class reunion in a support group setting would help reinforce keys to breastfeeding success and provide mother-to-mother sharing of challenges and advice.

\section{Optimizing Early Breastfeeding}

Selection of a birthing hospital that is baby friendly or a participant in the Baby-Friendly Hospital Initiative (BFHI) has been shown to increase breastfeeding initiation and duration. ${ }^{31}$ A survey of the nation's first 29 Baby-Friendly hospitals found the breastfeeding initiation rate at the BabyFriendly hospitals averaged $83.8 \%$ in 2001 , when the national average was $69.5 \%$. The mean exclusive breastfeeding rate at the Baby-Friendly hospitals was $78.4 \%$, compared with $46.3 \%$ nationwide. $^{32}$ Baby-Friendly hospitals are implementing the practices that are most supportive of breastfeeding detailed in the Ten Steps to Successful Breastfeeding sponsored by World Health Organization (WHO) and United Nation Children's Fund (UNICEF), and endorsed by the American Academy of Pediatrics (AAP) ${ }^{33}$ (Table 3). Each added step in the direction toward the Ten Steps has been shown to increase breastfeeding outcomes, demonstrating that changes toward the goals of the BFHI, all or in part, are successful. ${ }^{34,35}$ 
Table 3. Ten Steps To Successful Breastfeeding

The Baby-Friendly Hospital Initiative promotes, protects, and supports breastfeeding through the Ten Steps to Successful Breastfeeding for Hospitals, as outlined by United Nation Children's Fund and World Health Organization. The steps for the United States are:

1. Have a written breastfeeding policy that is routinely communicated to all healthcare staff.

2. Train all healthcare staff in skills necessary to implement this policy.

3. Inform all pregnant women about the benefits and management of breastfeeding.

4. Help mothers initiate breastfeeding within 1 hour of birth

5. Show mothers how to breastfeed and how to maintain lactation even if they are separated from their infants.

6. Give newborn infants no food or drink other than breastmilk unless medically indicated.

7. Practice rooming-in-allow mothers and infants to remain together 24 hours a day.

8. Encourage breastfeeding on demand.

9. Give no pacifiers or artificial nipples to breastfeeding infants.

10. Foster the establishment of breastfeeding support groups and refer mothers to them on discharge from the hospital or clinic.

Available at www.babyfriendlyusa.org/eng/10steps.html

Economically, a study in Texas demonstrated that the process of implementing the BFHI in U.S. acute care hospital settings is relatively cost-neutral. ${ }^{36}$

\section{Practical Tips on Breastfeeding Success}

Families who arrive for the birth of their infant with an educated intention to breastfeed will be more likely to succeed. Families who are confident in their decisions are more likely to make their breastfeeding desires known, request assistance, and avoid formula supplementation. Assuming a routine vaginal delivery, a stable newborn should be placed in skin-toskin contact with the mother, naked and prone on the mother's bare chest. The infant is dried, covered with a blanket, and allowed to transition in direct contact with the mother. This early bonding opportunity has been shown to assist the transitioning infant metabolically and thermodynamically. It also provides an early opportunity for introduction to the breast and promotes breastfeeding initiation within 1 hour of delivery. Early feedings provide the foundation and reinforce the expectation that babies should be breastfed early and often. Postcesarean birth babies will be encouraged to breastfeed as soon as possible, ideally in the operating room or recovery area. Vitamin $\mathrm{K}$ injection and prophylactic antibiotics to prevent ophthalmia neonatorum can be delayed for the first hour after birth to allow for facilitation of skin-to-skin contact and initiation of breastfeeding with the mother. ${ }^{37}$

Rooming-in, that is, having mother and infant in the same room, encourages more opportunities to breastfeed in a timely fashion. Close proximity allows for identification of early signals of hunger from the infant, such as sucking on the fist, rooting, or stirring in sleep. Identifying these early cues facilitates latching at the breast in a more controlled fashion for mother and baby. Hospital support staff, including delivery and postpartum nurses and lactation consultants, are a big part of breastfeeding success. Breastfeeding assessment, teaching, and documentation of feeds, voids, and stools should be done at every opportunity on each nursing shift. Allowing the family to particpate in logging of the data will help them continue their own diary of input and output upon discharge. This diaper diary record of information helps families keep on track with feedings and demonstrates evidence of increasing milk transfer with the growing number of voids and stools that should occur. It also provides great documentation for parents to bring to their first doctor visit. Additional knowledge and skills the breastfeeding mother should have before discharge are shown in Table 4.

\section{Postdischarge Follow-Up}

Supporters of breastfeeding have long advocated early follow-up visits within the first week of life, preferably within 48-72 hours of hospital discharge. The July 2004 AAP Clinical Practice Guideline, Management of hyperbilirubinemia in the newborn infant 35 or more weeks of gestation, ${ }^{38}$ lends additional weight to the need for early postdischarge follow-up. The guideline recommends that all infants be examined by a qualified healthcare professional within 48 hours of discharge to assess infant well-being and the presence of jaundice. The follow-up examination should include the infant's weight and percent change from birth weight, adequacy of intake (8-12 breastfeeds per day), pattern of voiding and stooling, observation for jaundice, and measurement of transcutaneous or serum bilirubin levels if indicated. The Academy of Breastfeeding Medicine Protocol, Guidelines for management of jaundice in the breastfeeding infant equal to or greater than 35 weeks' gestation, helps address medically indicated reasons for supplementation, such as clinical dehydration, weight loss $>8 \%-10 \%$, delayed bowel movements, significant hyperbilirubinemia, delayed maternal milk onset, or hypoglycemia. Supplementation with expressed breast milk is ideal, followed by pasteurized donor breast milk, and lastly, infant formula if necessary. ${ }^{39}$

\section{Maintaining Milk Supply}

Adequate milk supply is a concern of many mothers and healthcare professionals. Routine, effective milk removal from the breasts is essential to growing and maintaining an adequate supply. An active whey protein isolated from milk has been

Table 4. Before Hospital Discharge, Breastfeeding Mothers Should be Able to:

1. Position baby correctly at breast with no pain during feeding.

2. Latch the baby to breast properly.

3. Note when baby is swallowing milk.

4. State that the baby should be nursed a minimum of 8-12 times a day until satiety, with some infants needing to be fed more frequently.

5. State age-appropriate elimination patterns (at least six urinations per day and four stools per day by the fourth day of life).

6. List indications for calling healthcare professional. 7. Manually express milk from their breasts.

Adapted from Academy of Breastfeeding Medicine Clinical Protocol no. 7: Model Breastfeeding Policy. ${ }^{37}$ 
shown to act as a feedback inhibitor of lactation (FIL) when allowed to collect in the breast. The frequency or completeness of FIL removal regulates the rate of milk secretion by a mechanism that has been found to be local, chemical, and inhibitory in nature. Collection of the FIL in the breast inhibits milk constituent synthesis and decreases milk secretion on an autocrine basis. Therefore, completeness of milk removal (and FIL) ensures ongoing supply. ${ }^{40}$ Mothers should practice breast massage and compression during feedings to assist with maximal milk emptying. This concept is especially important for mothers who are separated from their infants or choose to use a breast pump for milk expression. A hands-on approach, incorporating manual massage and compression during pumping, will aid in the completeness of milk removal. ${ }^{41}$

\section{Early Barriers to Breastfeeding: Nipple Pain and Mastitis}

Nipple pain has been found to be one of the most common early breastfeeding challenges. For most women, this is a transitory discomfort that improves relatively quickly. However, complaints of nipple pain should prompt an evaluation of the infant's latch at the breast and perhaps repositioning of the infant. Often, a positional change that helps to extend rather than flex the baby's neck or gentle pressure on the chin of the infant to encourage a wider gape is a simple fix. A thorough examination of the infant's oral cavity may identify ankyloglossia or tongue-tie, which can affect tongue movement and function and cause nipple abrasion. For mild nipple soreness or cracking, applying expressed breast milk, warm water compresses, lanolin cream, or tea bag compresses has been shown to be equally effective. ${ }^{42}$

Prevention of the greater problem of mastitis and being on the lookout for signs of milk obstruction are key. The risk of mastitis can be minimized with unrestricted feeding and avoiding constriction from bra or purse strap and pressure from side lying, seatbelts, and so on. Mothers should take care to perform routine breast inspection and palpation during feedings to identify lumps, pain, or redness. If these symptoms are noted, particularly if the mother is experiencing fever, body aches or flu-like symptoms, she should contact her healthcare provider and rest, increase breastfeeding, and use hand expression, warm compresses, and massage to relieve milk stasis. Anti-inflammatory/analgesics, such as ibuprofen, are safe for breastfeeding mothers to relieve discomfort. A review of the treatment of mastitis, with suggestions for covering Staphylococcus, Streptococcus, and Escherichia coli, can be found in the Academy of Breastfeeding Medicine Protocol: Mastitis. ${ }^{43}$

\section{Mothers on Medications}

Mothers often have questions about over-the-counter or prescription medications and their impact on breastfeeding. There are very few medications that are contraindicated in breastfeeding. Helpful references to assist in counseling breastfeeding mothers include LactMed, a feature of TOXNET on the National Library of Medicine Website. ${ }^{44}$ Excellent reference books on the subject include Medications in Mother's Milk by Hale ${ }^{45}$ and Drugs in Pregnancy and Lactation by Briggs et al. ${ }^{46}$

\section{Help for Mothers in the Workplace}

More than half of mothers work outside the home before their children are 1 year old. Although $75 \%$ of new mothers start out breastfeeding, only $13 \%$ continue through their infant's first birthday. The federal 2010 Patient Protection and Affordable Care Act stipulates:

\begin{abstract}
An employer shall provide a reasonable break time for an employee to express breast milk for her nursing child for 1 year after the child's birth... a place, other than a bathroom, that is shielded from view and free from intrusion from co-workers and the public, which may be used by an employee to express breast milk. An employer shall not be required to compensate an employee receiving reasonable break time...for any work time spent for such purpose. ${ }^{\prime 47}$
\end{abstract}

The Business Case for Breastfeeding, a U.S. Department of Health and Human Services toolkit, is a comprehensive program designed to educate employers about the value of supporting breastfeeding employees in the workplace. ${ }^{48}$ The program offers tools to develop breastfeeding-friendly worksites and identify barriers that deter breastfeeding. Benefits to employers include lower healthcare costs, decreased absenteeism, and higher employee productivity, morale, and retention. The motto: retain instead of retrain is a financially sound and important one.

The January 2011, The Surgeon General's Call to Action to Support Breastfeeding, outlines steps that can be taken to remove some of the obstacles faced by women who want to breastfeed their babies. It also identifies ways that families, communities, employers, and healthcare professionals can improve breastfeeding rates and increase support for breastfeeding. ${ }^{49}$ In February 2011, the Internal Revenue Service ruled that mothers may use pretax money from their flexible spending accounts to cover the cost of breast pumps and other supplies. ${ }^{50}$

\section{Summary}

The majority of U.S. women are choosing to initiate breastfeeding in the hospital, but for many reasons, breastfeeding duration rates fall short of U.S. goals. Many barriers exist for mothers and babies to be successful in the short-term and long-term aspects of the first year. As healthcare providers aware of the mounting evidence that breastfeeding is good for mothers, babies, families, and society, we should begin to educate our families earlier and more often that breastfeeding can make a difference in their health. Recognizing that breastfeeding requires support on many fronts-hospitals, home environments, communities, and the workplace-we can help families take advantage of the new programs of support. Best practices for breastfeeding should be recognized, promoted, rewarded, and publicized to increase public awareness and acceptance.

\section{Disclosure Statement}

The authors have no conflicts of interest to report.

\section{References}

1. Centers for Disease Control and Prevention. National Immunization Survey, provisional data, 2007 births. Available at www.cdc.gov/breastfeeding/data/NIS_data/index.htm

2. Morrow AL, Ruiz-Palacios GM, Jiang X, et al. Human-milk glycans that inhibit pathogen binding protect breast-feeding infants against infectious diarrhea. J Nutr 2005;135:1304-1307.

3. Mahmud MA, Chappell CL, Hossain MM, et al. Impact of breast-feeding on Giardia lamblia infections in Bilbeis, Egypt. Am J Trop Med Hyg 2001;65:257-260. 
4. Chompook P, Todd J, Wheeler JG, et al. Risk factors for shigellosis in Thailand. Int J Infect Dis 2006;10:425-433.

5. Chak E, Rutherford GW, Steinmaus C. The role of breastfeeding in the prevention of Helicobacter pylori infection: A systematic review. Clin Infect Dis 2009;48:430-437.

6. Quigley MA, Kelly YJ, Sacker A. Breastfeeding and hospitalization for diarrheal and respiratory infection in the United Kingdom. Millennium Cohort Study. Pediatrics 2007;119: e837-e842.

7. Sabirov A, Casey JR, Murphy TF, et al. Breast-feeding is associated with a reduced frequency of acute otitis media and high serum antibody levels against NTHi and outer membrane protein vaccine antigen candidate P6. Pediatr Res 2009;66:565-570.

8. Duijts L, Jaddoe V, Hofman A, Moll H. Prolonged and exclusive breastfeeding reduces the risk of infectious diseases in infancy. Pediatrics 2010;126:e18-e25.

9. Levy I, Comarsca J, Davidovits M, et al. Urinary tract infection in preterm infants: The protective role of breastfeeding. Pediatr Nephrol 2009;24:527-531.

10. Hamosh M, Peterson JA, Henderson TR, et al. Protective function of human milk: The milk fat globule. Semin Perinatol 1999;23:242-249.

11. Newburg DS, Peterson JA, Ruiz-Palacios GM, et al. Role of human-milk lactadherin in protection against symptomatic rotavirus infection. Lancet 1998; 18;351:1160-1164.

12. Garofalo R. Cytokines in human milk J Pediatr 2010; 156(Suppl 2):S36-40.

13. Sela DA, Chapman J, Adeuya A, et al. The genome sequence of Bifidobacterium longum subsp. infantis reveals adaptations for milk utilization within the infant microbiome. Proc Natl Acad Sci USA 2008;105:18964-18969.

14. Ip S, Chung M, Raman G, Chew P, Magula N, DeVine D, Trikalinos T, Lau J. Breastfeeding and maternal and infant health outcomes in developed countries. Evid Rep Technol Assess 2007;153:1-186.

15. Schwarz EB, Ray RM, Stuebe AM, et al. Duration of lactation and risk factors for maternal cardiovascular disease. Obstet Gynecol 2009;113:974-982.

16. Mennella JA, Jagnow CP, Beauchamp GK. Prenatal and postnatal flavor learning by human infants. Pediatrics 2001;107:E88.

17. Doucet S, Soussignan R, Sagot P, et al. The secretion of areolar (Montgomery's) glands from lactating women elicits selective, unconditional responses in neonates. PLoS One 2009;4:e7579.

18. Schaal B, Coureaud G, Doucet S, et al. Mammary olfactory signalisation in females and odor processing in neonates: Ways evolved by rabbits and humans. Behav Brain Res 2009;200:346-358.

19. Doucet $S$, Soussignan R, Sagot $P$, et al. The "smellscape" of mother's breast: Effects of odor masking and selective unmasking on neonatal arousal, oral, and visual responses. Dev Psychobiol 2007;49:129-138.

20. Spencer NA, McClintock MK, Sellergren SA, et al. Social chemosignals from breastfeeding women increase sexual motivation. Horm Behav 2004;46:362-370.

21. The economic benefits of breastfeeding: A review and analysis. USDA food assistance and nutrition research report no. 13, March 2001.

22. Bartick M, Reinhold A. The burden of suboptimal breastfeeding in the United States: A pediatric cost analysis. Pediatrics 2010;125;e1048-e1056.
23. Ball TM, Wright AL. Health care costs of formula feeding in the first year of life. Pediatrics 1999;103:870-876.

24. Oliveira V, Prell M, Smallwood D, et al. WIC and the retail price of infant formula. Food Assistance and Nutrition Research Report No. FANRR 39 1, 2005. Available at www.ers .usda.gov/Publications/FANRR39 1/FANRR39 1.pdf

25. Oliveira V, Frazao E. The WIC program: Background, trends, and economic issues, 2009 ed. Available at www .ers.usda.gov/Publications/ERR73/ERR73.pdf

26. Arora S, McJunkin C, Wehrer J, Kuhn P. Major factors influencing breastfeeding rates: Mother's perception of father's attitude and milk supply. Pediatrics 2000;106:e67.

27. Kessler LA, Gielen AC, Diener-West M, Paige DM. The effect of a woman's significant other on her breastfeeding decision. J Hum Lact 1995;11:103-109.

28. www.bestforbabes.org

29. U.S. Preventative Services Task Force Recommendation Statement. Primary care interventions to promote breastfeeding. Ann Intern Med 2008;149:560-564.

30. Dyson L, McCormick FM, Renfrew MJ. Interventions for promoting the initiation of breastfeeding. Cochrane Database of Systematic Reviews 2005, Issue 2. Art. No. CD001688.

31. Philipp BL, Merewood A, Miller LW, et al. Baby-Friendly Hospital Initiative improves breastfeeding initiation rates in a U.S. hospital setting. Pediatrics 2001;108:677-681.

32. Merewood A, Mehta SD, Chamberlain LB, Philipp BL, Bauchner H. Breastfeeding rates in U.S. Baby-Friendly hospitals: Results of a nationwide survey. Pediatrics 2005;116: 628-634.

33. Baby-Friendly USA, Inc. The ten steps to successful breastfeeding. Available at www.babyfriendlyusa.org/eng/10steps .html

34. Fairbank L, O'Meara S, Renfrew MJ, Woolridge M, Snowden AJ, Lister-Sharp D. A systematic review to evaluate the effectiveness of interventions to promote the initiation of breastfeeding. Health Technol Assess 2000;4:1-171.

35. DiGirolamo AM, Grummer-Strawn LM, Fein S. Effect of maternity care practices on breastfeeding. Pediatrics 2008; 122(Suppl 2):S43-S49.

36. DelliFraine J, Langabeer J 2nd, Williams JF, Gong AK, Delgado RI, Gill SL. Cost comparison of Baby Friendly and nonBaby Friendly hospitals in the United States. Pediatrics 2011;127:e989-994.

37. Academy of Breastfeeding Medicine Clinical Protocol no. 7: Model breastfeeding policy. Breastfeed Med 2010:5;173-177.

38. AAP clinical practice guideline: Management of hyperbilirubinemia in the newborn infant 35 or more weeks of gestation. Pediatrics 2004;114:297-316.

39. ABM clinical protocol no. 22: Guidelines for management of jaundice in the breastfeeding infant equal to or greater than 35 weeks' gestation. Breastfeeding Med 2010;5:87-93.

40. Wilde CJ, Addey CV, Boddy LM, Peaker M. Autocrine regulation of milk secretion by a protein in milk. Biochem Soc Symp 1998;63:81-90.

41. Morton J, Hall JY, Wong RJ, Thairu L, Benitz WE, Rhine WD. Combining hand techniques with electric pumping increases milk production in mothers of preterm infants. J Perinatol 2009;29:757-764.

42. Lochner JE, Livingston CJ. Which interventions are best for alleviating nipple pain in nursing mothers? J Fam Pract 2009;58:612a-612c.

43. Academy of Breastfeeding Medicine Clinical Protocol no. 4: Mastitis. Breastfeeding Med 2008;3:177-180. 
44. Lactmed. Drugs and Lactation Database Available at toxnet.nlm.nih.gov

45. Hale T. Medications and mother's milk: A manual of lactational pharmacology, 11th ed. Amarillo, TX: Pharmasoft Medical Pub, 2010.

46. Briggs GG, Freeman RK, Yaffe SJ. Drugs in pregnancy and lactation: A reference guide to fetal and neonatal risk. Philadelphia: Lippincott Williams \& Wilkins, 2008.

47. www.ncsl.org/IssuesResearch/Health/BreastfeedingLaws/ tabid/14389/Default.aspx

48. www.womenshealth.gov/breastfeeding/government-programs/business-case-for-breastfeeding/
49. www.surgeongeneral.gov/topics/breastfeeding/

50. www.nytimes.com/2011/02/11/business/11breast.html

Address correspondence to: Michelle G. Brenner, M.D., IBCLC Eastern Virginia Medical School, Pediatrics 601 Children's Lane Norfolk, VA 23507

E-mail: michelle.brenner@gmail.com 
